\title{
Distribution, Relative Abundance, Species Diversity and Richness of Syrphid Flies in Floricultural Ecosystem of Kashmir, India
}

\author{
Akhtar Ali Khan* \\ Division of Entomology, Sher-e-Kashmir University of Agricultural Sciences and Technology of \\ Kashmir, Shalimar, Srinagar-190025, Jammu and Kashmir, India \\ *Corresponding author
}

\section{A B S T R A C T}

\begin{tabular}{|l|}
\hline K e y w or d s \\
Biodiversity, \\
distribution, \\
Relative abundance, \\
Species diversity, \\
Species richness, \\
Syrphid fly, \\
Floriculture \\
\hline Article Info \\
\hline Accepted: \\
19 July 2017 \\
Available Online: \\
10 September 2017 \\
\hline
\end{tabular}

The extensive study on biodiversity of aphidophagous syrphid flies were made in daffodil, Rose, chrysanthemum and wild flowers (including Dandelion, Fennel and Black Berry) of floriculture ecosystem of Kashmir during 2013 to 2014 and a total of 21 species were recorded. Among all, the Eristalis tenax (11.57\% and 9.80\%) was found most distributed and abundant syrphid species followed by Eoseristalis cerealis $(10.49 \%$ and $9.00 \%)$ and Eupeodus corolla (9.12\% and $7.63 \%)$ while as Syritta sp. (0.82\% and $1.17 \%)$ followed by Palpada sp. (1.49\% and 2.02\%) were least distributed and abundant species of syrphid fly in daffodil, Rose, chrysanthemum and wild flowers (including Dandelion, Fennel and Black Berry) of floricultural ecosystem of all locations in Kashmir during 2013 and 2014, respectively. The species diversity was measured by the using Shannon-Wiener biodiversity index $(\mathrm{H})$ and species richness by using Margalef's richness index (Da) during 2013 and 2014. The highest mean species diversity $\left(\mathrm{H}^{!}\right)$of syrphid flies was found in Harwan of district Srinagar with 2.935 and the highest species richness (Da) was observed in Mamoosa of district Baramullah with 3.584 and 3.611 in Shalimar of district Srinagar of floricultural ecosystem of Kashmir.

\section{Introduction}

Flies are common flower visitors. Species from at least 86 families of Diptera have been observed visiting flowers, and over eleven hundred species of plants from 172 families have been reported as having their flowers visited by flies (Kearns, 2002; Khan and Riyaz, 2017; this is undoubtedly an underestimate of the actual number). The systematic study of the family Syrphidae has gradually progressed from the 17th century and presently 6,107 species under 209 genera reported from the world (Pape and Evenhuis, 2013, Miranda et al., 2013). In India, 357 species under 14 tribes of three subfamilies are reported (Ghorpade, 2014; Mitra et al., 2015).

Syrphinae and Milesiinae are the two most important sub families of the family Syrphidae. Most of the hoverflies are important pollinators of the many flowers. Larval stage of the many species of the hoverflies is also predator of the aphid and some other pests (Gilbert, 1981; Khan et al., 2016; Khan, 2017; Khan and Riyaz, 2017). Larvae of hoverflies have different feeding modes like saprophagous, aphidophagous, zoophagous and phytophagous (Sommaggio, 
1999). While the adults are the flower visitors they can visit large number of flowers in one day. Adult hoverflies feed on plant nectar for energy while the requirement of protein is fulfilled by feeding on pollens (Mitra et al., 2005; 2008). Nector and pollen also increases their egg laying capacity and life of the adult dipterans (Khan and Hanif, 2016). Some of the hoverflies species (Syrphidae) are also considered as very important pollinators of different crops.

Pollination and biological control are one of the most important ecosystem services delivered by insects for human beings (Potts et al., 2006, Khan et al., 2016, Khan, 2017a,b,). Both the abundance and diversity of pollinators have generally been documented as beneficial to the yield of numerous crops (Talavera et al., 2001; Kremen et al., 2002). However, there has been relatively little research on the flower flies pollination compared with bee species (Mitra et al., 2005, 2010 and 2013). Bees is the only taxon which is considered thoroughly in this context (Jauker and Wolters, 2008) and very little work has been done on fly (Diptera) pollinators (Ssymank et al., 2008).

Flower flies are a very important group of insects because their services to ecosystems are twofold i.e., their larvae are important natural enemies of herbivorous arthropods and their adults are pollinators of many of the crops and wild plants (Tooker et al., 2006; Ghahari et al., 2008).

It is estimated that their importance as predator is equal to that of parasitoids, pathogenic fungi, lady birds and lacewings (Ankersmit et al., 1986). Adults of hover flies consume floral nectar for energy and pollen for protein, lipids and vitamins (Faegri and Pijl, 1979). These floral resources enhance longevity and fecundity of adult hoverflies (Shahjhan, 1968). This nutritional dependence on floral resources suggests that artificial planting of the most attractive nutrition plants, might help to conserve hover flies for the better biological control of herbivorous pests (Heimpel and Jervis, 2005). Colley and Luna (2000) called it "beneficial insectary planting" when we intentionally introduce flowering plants into agricultural ecosystems to increase pollen and nectar resources required by Syrphids. Therefore, the effective biological control by using beneficial insectary plants needs a complete knowledge of floral host range of biological control agents (Colley and Luna, 2000; Tooker et al., 2006). Furthermore, most of the investigations of predator foraging have been made taking into account prey-host-relationships exclusively and have overlooked non-prey food (Jervis and Kidd, 1996). The aim of present study, explore the distribution, relative abundance, species diversity and richness of syrphid flies for utilization as pollinator and biological control agents in floriculture ecosystem of Kashmir

\section{Materials and Methods}

\section{Study area}

In floricultural ecosystem, biodiversity of syrphid flies were studied in two district and three respective locations viz., Srinagar (Shalimar, Harwan and Gulabagh) and Baramullah (Wagoora, Mamoosa and Warpora) of Kashmir during 2013 and 2014. In each location, four flower crops viz., daffodil, Rose, chrysanthemum and wild flowers (including Dandelion, Fennel and Black Berry) were chosen for study of distribution, relative abundance, species diversity and species richness in floricultural ecosystem of Kashmir during 2013 and 2014.

\section{Method of sampling}

Two sampling methods were used, the detail as follows: 


\section{Sweep net}

In sweep net method, adult syrphids were collected by making double stroke sweeps by insect collection net (Diameter $32 \mathrm{~cm}$ and handle $92 \mathrm{~cm}$ ) was repeated five times randomly from five different places of floriculture ecosystem.

\section{Hand picking}

The maggots (larvae) populations were recorded on the basis of population per plant. The maggots were collected into plastic tube by hand picking method.

\section{Preservation and identification}

The collected samples were identified upto the species level with the help of literature and confirm by experts.

The larvae were reared up to adult stage in separate labelled rearing cages by providing fresh aphids every day. After adult emergence, the adults were preserved dry and grouped into different categories based on morphological features and counted.

\section{Statistical analysis}

\section{Ecological indices for quantitative analysis}

Quantitative estimation of individual species in floriculture ecosystem was made using the data derived from field survey. Species richness (Da) was calculated using Margalef's richness index and species diversity was calculated by using Shannon-Wiener diversity index by using the data.

\section{Margalef's richness index}

The simplest measure of species diversity is the number of species or species richness and was calculated after Margalef's (1968).
$\mathrm{Da}=(\mathrm{S}-1) / \log$ e $\mathrm{N}$

Where, $D a=$ Margalef's richness index, $\mathrm{S}=$ Number of species, and $\mathrm{N}=$ total number of individuals.

\section{Shannon-Wiener diversity index}

The Shannon Wiener diversity index (Shannon, 1948) provides a good learning tool for computing two distinct habitats of floriculture of Kashmir. It combines two quantifiable measures, the species richness (the number of species within the community) and the species equitability (how even are the number of individual species). It is computed by using following equation;

$\mathrm{H}=-\sum \mathrm{pi} \ln \mathrm{pi}$

Where,

$\mathrm{H}=$ Shannon-Wiener diversity index

$\mathrm{Pi}=$ the observed proportion of a particular species.

The value of $\mathrm{H}$ near zero would indicate that every species in the sample is the same. A value near 4.6 would indicate that the numbers of individuals are evenly distributed between all the species. Values in the middle are ambiguous which an obvious flaw of this index is and, thus, care is taken when using this index.

\section{Results and Discussion}

\section{Distribution of syrphid fly}

In floricultural ecosystem, 21 species of syrphid flies were recorded during 2013. Out of 21 species, 16, 17, 15, and 21;19, 18, 16 and $21 ; 9,10,14$ and 21 species were recorded from Daffodil, rose, chrysanthemum and wild flowers in Shalimar, Harwan and Gulabagh 
respectively of district Srinagar while as 9, 12,17 and 19;12, 12, 11 and 21;10, 9, 13 and 19 were recorded from Daffodil, rose, chrysanthemum and wild flowers in Wagoora, Mamoosa and Warpora respectively of district Baramullah during 2013 (Table 1). Among all species, Eristalis tenax was the most distributed species followed by Eoseristalis cerealis, Eupeodus corolla and Eristalis interruptus were recorded during 2013 in floricultural ecosystem of Kashmir. Species of subfamily- Eristalinae; Eristalis tenax, Eoseristalis cerealis and Eristalis interruptus were found most distributed species of syrphid fly while as Syritta sp., Palpada sp. and Eristalinus sp. were least distributed species and other species were more or less evenly distributed in floricultural ecosystem of Kashmir during 2013. In subfamilySyrphinae; Eupeodus corolla, Sphaerophoria scripta and Sphaerophoria bengalensis were most abundant distributed species of syrphid flies while as Syrphus sp., Scaeva pyrastri and Epistrophe grossularia were found least distributed in floricultural ecosystem of Kashmir during 2013.

In 2014, Out of 21 species, 17, 15, 18, and 19; $14,15,11$ and 21; 16, 13,13 and 19 species were recorded from Daffodil, rose, chrysanthemum and wild flowers in Shalimar, Harwan and Gulabagh respectively of district Srinagar while as 14, 16,15 and 19; 14, 13, 11 and 18; 12, 14, 15 and 18 species were recorded from Daffodil, rose, chrysanthemum and wild flowers in Wagoora, Mamoosa and Warpora respectively of district Baramullah during 2014 in floricultural ecosystem of Kashmir (Table 2). Among all species, Eristalis tenax followed by Eoseristalis cerealis, Eupeodus corolla and Sphaerophoria scripta were found most distributed while as Syritta sp., Palpada sp. and Eristalinus sp. were least distributed species and other species were more or less evenly distributed in floricultural ecosystem of Kashmir during 2014. In sub- familyEristalinae: Eristalis tenax followed by Eoseristalis cerealis, Eristalis interruptus and Eristalinus aeneus were found most distributed while as Syritta sp., Palpada sp. an d Eristalinus sp. were least distributed species in floricultural ecosystem of Kashmir during 2014. In sub-family - Syrphinae; Eupeodus corolla followed by Sphaerophoria scripta and Sphaerophoria bengalensis were most abundant distributed species of syrphid flies while as Syrphus sp., Scaeva pyrastri and Epistrophe grossularia were found least distributed in floricultural ecosystem of Kashmir during 2014.

Mitra et al., 2015 was review and recorded 357 species of hoverflies, more than 100 species are restricted in India out of which $22.13 \%$ represented the Jammu and Kashmir. Khan and Hanif (2016) reported 16 species from Chakwal, Pakistan and Khan and Riyaz (2017) reported 19 species of syrphid flies from fruit orchards of Kashmir and these findings has quite similar to our findings. In present study the Eristalis tenax followed by Eoseristalis cerealis, Eristalis interruptus and Eristalinus aeneus were found most distributed syrphid fly species. According to Khan and Riyaz (2017) the distribution of Eristalis tenax followed by Eoseristalis cerealis, Eristalis interruptus and Eristalinus balteatus was found quit similar to present findings and variations were due to climatic variation and ecology of area (Ssmank et al., 2008; Sajjad et al., 2010)

\section{Relative abundance of syrphid flies}

In floricultural ecosystem, among 21 species, highest mean population of E. tenax $(11.57 \%)$ followed by Eoseristalis cerealis (10.49\%), Eupeodus corolla (9.12\%), and E. aeneus $(7.56 \%)$ were recorded in floricultural ecosystem of Kashmir during 2013. Least population $(0.82 \%)$ of Syritta $s p$ followed by 
Palpada sp (1.49\%) and Eristalinus sp. $(1.52 \%)$ were recorded in floricultural ecosystem during 2013. The population of $E$. tenax was recorded highest in almost all floricultural crops including Daffodil, rose, chrysanthemum and wild flowers in different location of district Srinagar and Baramullah. Among all floricultural crops, the syrphid flies were observed highest in wild flowers followed by Rose crop in floricultural ecosystem of Kashmir during 2013. The mean population of syrphid flies were taken from the Daffodil, rose, chrysanthemum and wild flowers crops in two districts with three respective locations viz., Srinagar (Shalimar, Harwan and Gulabagh) and Baramullah (Wagoora, Mamoosa and Warpora) of floricultural ecosystem of Kashmir during 2013. The population of E. tenax was recorded highest in almost all floricultural crops including Daffodil, rose, chrysanthemum and wild flowers crops in different location of district Shalimar and Baramullah (Table 3).

Out of two sub-families, the abundance of Eristalinae was higher than the Syrphidae in all locations, flowers and districts during 2013. In sub-family Eristalinae, the most abundant species was Eristalis tenax $(11.57 \%)$ followed by Eoseristalis cerealis $(10.49 \%)$ while as Syritta sp. $(0.82 \%)$ and Palpada sp. (1.49\%) were found least abundant and under the sub-family: Syrphinae; Eupeodus corolla (9.12\%) followed by Sphaerophoria scripta (6.42\%) and Sphaerophora bengalensis $(5.05 \%)$ were recorded abundant while as Syrphus sp. (2.47\%), Scaeva pyrastri (2.38\%) and Epistrophe grossularia $(4.03 \%)$ were found least abundant in floricultural ecosystem of Kashmir during 2013 (Table 3).

In 2014, the highest abundance species was also E. tenax $(9.80 \%)$ followed by Eoseristalis cerealis (9.00\%), Eupeodus corolla (7.63\%), and Sphaerophoria scripta $(7.10 \%)$ were recorded in floricultural ecosystem of Kashmir during 2014. Least population $(1.17 \%)$ of Syritta sp followed by Palpada sp (2.02\%) and Helophilus sp. $(2.10 \%)$ were recorded in floricultural ecosystem during 2014.

Out of total 21 species, most of the species of syrphid fly was recorded in wild flowers of all locations during 2014, which was quite similar to 2013. In sub-family- Eristalinae, the highest abundant species was Eristalis tenax $(9.80 \%)$ followed by Eoseristalis cerealis (9.00\%), E. interruptes $(6.71 \%)$ and while as least abundant species was Syritta sp. (1.17\%) followed by Palpada sp. (2.02\%) and Helophilus sp. (2.10\%).

In subfamily- Syrphinae, the highest abundant species were Eupeodus corolla (7.63\%) followed by Sphaerophora scripta (7.10\%) and Sphaerophora bengalensis (5.52\%) in floricultural ecosystem of Kashmir during 2014.

During both years, the Eristalis tenax was found most abundant syrphid species followed by Eoseristalis cerealis and Eupeodus corolla while as Syritta sp. followed by Palpada sp. were least abundant species of syrphid fly in floricultural ecosystem of Kashmir (Tables 3 and 4). Syrphids are generally selective of the floral resources that they utilize, but some species are more specialized than others, and males are more selective than females (Gilbert 1981; Shaw and Taylor 1986; Haslett 1989b; Cowgill et al., 1993). Large, hairy flower flies such as Eristalis tenax L. and Lasiopticus [Scaeva] pyrastri L. often contain pollen of numerous plant species in their crops (Holloway, 1976), so they appear to exhibit little flower constancy, although their migratory behaviour could be responsible for some of this diversity and abundance. 
Table.1 Distribution of Syrphid flies in major flowers of Kashmir during 2013

\begin{tabular}{|c|c|c|c|c|c|c|c|c|c|c|c|c|c|c|c|c|c|c|c|c|c|c|c|c|c|}
\hline \multirow{4}{*}{ Sub-family, species } & \multicolumn{24}{|c|}{ Distribution of Syrphid flies in Floriculture Ecosystem of Kashmir (in number) } & \multirow{4}{*}{ Total } \\
\hline & \multicolumn{12}{|c|}{ Srinagar } & \multicolumn{12}{|c|}{ Baramullah } & \\
\hline & \multicolumn{4}{|c|}{ Shalimar } & \multicolumn{4}{|c|}{ Harwan } & \multicolumn{4}{|c|}{ Gulabagh } & \multicolumn{4}{|c|}{ Wagoora } & \multicolumn{4}{|c|}{ Mamoosa } & \multicolumn{4}{|c|}{ Warpora } & \\
\hline & Daff. & Ro & Chry & WFs & Daff. & Ro & Chry & WFs & Daff. & Ro & Chry & WFs & Daff. & Ro & Chry & WFs & Daff. & Ro & Chry & WFs & Daff. & Ro & Chry & WFs & \\
\hline \multicolumn{26}{|l|}{ Sub-Family: Eristalinae } \\
\hline Eristalinus taeniops (Wied.) & 5 & - & - & 5 & 4 & 3 & 2 & 6 & 3 & - & - & 2 & 4 & 2 & 2 & - & 2 & - & - & 4 & - & - & 3 & 3 & 50 \\
\hline Eristalinu sparia Bigot & 3 & 2 & - & 5 & 3 & - & 2 & 3 & - & - & - & 4 & - & - & 3 & 4 & 3 & - & 2 & 4 & - & - & - & 5 & 43 \\
\hline Eristalinus aeneus (Scopoli) & 6 & 4 & 6 & 10 & 7 & 8 & 4 & 9 & 4 & 6 & 3 & 8 & 2 & 5 & 3 & 8 & - & - & 5 & 10 & 3 & 3 & 6 & 10 & 130 \\
\hline Eristalinus sp. & 2 & 2 & - & 6 & 2 & 3 & 3 & 5 & - & - & - & 3 & - & - & - & 4 & 2 & - & - & 4 & - & - & - & - & 36 \\
\hline Eristalis arbustorum (L.) & 5 & 4 & 3 & 6 & 3 & 2 & - & 7 & - & - & 3 & 4 & - & 4 & - & 6 & 3 & 2 & - & 5 & 4 & - & 6 & 8 & 75 \\
\hline $\begin{array}{ll}\begin{array}{l}\text { Eoseristalis } \\
\text { (Fabicius) }\end{array} & \text { cerealis } \\
\end{array}$ & 8 & 7 & 6 & 15 & 6 & 9 & 5 & 15 & 9 & 6 & 5 & 12 & 7 & 4 & 5 & 11 & 6 & 5 & 7 & 9 & - & 4 & 6 & 13 & 180 \\
\hline Eristalis tenax (Linnaeus) & 4 & 9 & 8 & 15 & 5 & 7 & 7 & 12 & - & 9 & 8 & 14 & 8 & 9 & 6 & 14 & 3 & 9 & 8 & 13 & 4 & 8 & 6 & 15 & 201 \\
\hline Eristalis interruptus (Poda) & 3 & 5 & 7 & 15 & 2 & 5 & 3 & 16 & - & - & 2 & 13 & - & 8 & 7 & 11 & - & 5 & 9 & 14 & 2 & 4 & 7 & 13 & 151 \\
\hline Eristalis $\mathrm{sp}$. & 2 & 4 & - & 6 & 3 & - & - & 9 & 2 & 2 & - & 5 & - & - & 3 & 6 & - & 3 & 2 & 5 & 2 & 3 & - & 9 & 66 \\
\hline Palpada sp. & - & - & 2 & 4 & 2 & 2 & - & 5 & - & - & - & 4 & 2 & - & 2 & - & - & - & - & 5 & 2 & - & - & 3 & 33 \\
\hline Helophilus trivittatusWeid & 3 & 6 & 5 & 10 & 4 & 6 & 3 & 8 & 2 & 7 & 5 & 11 & - & 5 & 6 & 8 & 3 & - & - & 6 & 5 & 7 & 3 & 12 & 125 \\
\hline Helophilus sp. & - & - & - & 5 & - & 2 & 3 & 6 & - & - & 3 & 6 & - & - & - & 5 & - & - & 2 & 5 & - & - & - & 6 & 43 \\
\hline Syritta sp. & - & - & - & 5 & - & 2 & - & 6 & - & - & 2 & 4 & - & - & - & 4 & - & - & - & 3 & - & - & - & - & 26 \\
\hline Sub total & 41 & 43 & 37 & 107 & 41 & 49 & 32 & 107 & 20 & 30 & 31 & 90 & 23 & 37 & 37 & 81 & 22 & 24 & 35 & 87 & 22 & 29 & 37 & 97 & 1159 \\
\hline \multicolumn{26}{|l|}{ Sub-Family:Syrphinae } \\
\hline $\begin{array}{l}\text { Epistrophe } \\
\text { (Meigen) }\end{array}$ & 3 & 5 & 4 & 8 & 2 & 4 & 2 & 7 & - & 5 & 4 & 7 & 3 & 2 & 2 & 5 & - & - & 2 & 5 & 2 & - & 2 & 4 & 78 \\
\hline Episyrphusbalteatus(De Geer) & 3 & 6 & 5 & 10 & 2 & 5 & 4 & 9 & - & 3 & 2 & 13 & - & 5 & 5 & 8 & 2 & 5 & - & 10 & - & - & 2 & 4 & 103 \\
\hline Eupeodu s corolla (Fabricius) & 7 & 9 & 8 & 12 & 6 & 7 & 8 & 15 & 3 & 4 & 2 & 13 & 5 & - & 7 & 14 & 5 & 4 & 3 & 7 & 5 & 7 & 2 & 10 & 163 \\
\hline Scaeva pyrastri (Linneaus) & - & 4 & 3 & 7 & 2 & - & - & 6 & 3 & - & - & 5 & - & - & 3 & 6 & 2 & 2 & - & 5 & - & - & - & 6 & 54 \\
\hline $\begin{array}{l}\text { Sphaerophoria bengalensis } \\
\text { Mac. }\end{array}$ & 5 & 3 & 4 & 12 & 3 & 2 & 2 & 10 & 2 & 2 & - & 10 & 3 & 4 & 3 & 9 & - & 3 & 4 & 7 & - & 3 & 2 & 13 & 106 \\
\hline Sphaerophoria indiana Bigot & - & 4 & 5 & 12 & 3 & 2 & 6 & 13 & 4 & - & 3 & 8 & - & 4 & 2 & 8 & - & 2 & - & 4 & - & 3 & 2 & 9 & 94 \\
\hline $\begin{array}{ll}\begin{array}{l}\text { Sphaerophoria } \\
\text { (Linnaeus) }\end{array} & \text { scripta } \\
\end{array}$ & 5 & 6 & 5 & 13 & 4 & 3 & 5 & 10 & - & 4 & 9 & 12 & 4 & 3 & 8 & 11 & 2 & 3 & 2 & 7 & - & - & 4 & 9 & 129 \\
\hline Syrphus sp. & - & 3 & 3 & 6 & 3 & 2 & - & 5 & - & - & 2 & 5 & - & - & 2 & 4 & 3 & 2 & - & 6 & 2 & - & - & 4 & 52 \\
\hline Sub total & 23 & 40 & 37 & 80 & 25 & 25 & 27 & 75 & 12 & 18 & 22 & 73 & 15 & 18 & 32 & 65 & 14 & 21 & 11 & 51 & 9 & 13 & 14 & 59 & 779 \\
\hline Total individuals & 64 & 83 & 74 & 187 & 66 & 74 & 59 & 182 & 32 & 48 & 53 & 163 & 38 & 55 & 69 & 146 & 36 & 45 & 46 & 138 & 31 & 42 & 51 & 156 & 1938 \\
\hline Total species & 15 & 17 & 15 & 21 & 19 & 18 & 15 & 21 & 9 & 10 & 14 & 21 & 9 & 12 & 17 & 19 & 12 & 12 & 11 & 21 & 10 & 9 & 13 & 19 & 21 \\
\hline
\end{tabular}

Daff = Daffodil, Ro= Rose, Chry = Chrysanthemum, WFs= Wild flowers, Number of samples=120 sweeps in each flower crops of each locations, Sampling method=sweep net method, Sampling time=April to September at weekly interval 
Table.2 Distribution of Syrphid flies in major flowers of Kashmir during 2014

\begin{tabular}{|c|c|c|c|c|c|c|c|c|c|c|c|c|c|c|c|c|c|c|c|c|c|c|c|c|c|}
\hline \multirow{4}{*}{ Sub-family, species } & \multicolumn{24}{|c|}{ Distribution of Syrphid flies in major flowers of Kashmir (in number) } & \multirow{4}{*}{ Total } \\
\hline & \multicolumn{12}{|c|}{ Srinagar } & \multicolumn{12}{|c|}{ Baramullah } & \\
\hline & \multicolumn{4}{|c|}{ Shalimar } & \multicolumn{4}{|c|}{ Harwan } & \multicolumn{4}{|c|}{ Gulabagh } & \multicolumn{4}{|c|}{ Wagoora } & \multicolumn{4}{|c|}{ Mamoosa } & \multicolumn{4}{|c|}{ Warpora } & \\
\hline & Daff. & Ro & Chry & WFs & Daff. & Ro & Chry & WFs & Daff. & Ro & Chry & WFs & Daff. & Ro & Chry & WFs & Daff. & Ro & Chry & WFs & Daff. & Ro & Chry & WFs & \\
\hline \multicolumn{26}{|l|}{ Sub-Family: Eristalinae } \\
\hline Eristalinus taeniops (Wied.) & - & 4 & 2 & 3 & 5 & 2 & - & 3 & 3 & 2 & - & - & 3 & - & - & 3 & 6 & 4 & 3 & - & 2 & 2 & - & 2 & 49 \\
\hline Eristalinus paria Bigot & 2 & 2 & 3 & - & 4 & - & - & 3 & 5 & 4 & - & 2 & 4 & - & 3 & 2 & 3 & 2 & - & - & 4 & - & 2 & - & 45 \\
\hline Eristalinus aeneus (Scopoli) & 4 & 3 & 4 & 8 & 6 & 5 & 3 & 12 & 3 & 4 & 3 & 8 & 7 & 3 & 4 & 10 & 3 & 4 & 3 & 12 & - & 6 & 3 & 9 & 127 \\
\hline Eristalinus sp. & - & - & 2 & - & 4 & 3 & - & 2 & 3 & - & 4 & 2 & 2 & 2 & - & 2 & 2 & 2 & 4 & 2 & - & 4 & 3 & - & 43 \\
\hline Eristalis arbustorum (L.) & 6 & 3 & 4 & 3 & 4 & 3 & 2 & 5 & 3 & 2 & - & 2 & 4 & 2 & 3 & - & - & 4 & - & 5 & 2 & 4 & 3 & 4 & 68 \\
\hline $\begin{array}{ll}\begin{array}{l}\text { Eoseristalis } \\
\text { (Fabicius) }\end{array} & \text { cerealis } \\
\end{array}$ & 9 & 5 & 4 & 13 & 7 & 6 & - & 15 & 8 & - & 8 & 10 & 8 & 9 & 5 & 12 & - & 8 & 6 & 14 & 7 & 5 & 6 & 11 & 176 \\
\hline Eristalis tenax (Linnaeus) & 6 & 4 & 7 & 15 & 7 & 7 & 4 & 10 & 6 & 8 & 5 & 13 & 7 & 6 & 5 & 13 & 5 & 6 & 4 & 13 & 8 & 9 & 6 & 14 & 188 \\
\hline Eristalis interruptus (Poda) & - & 5 & 6 & 8 & - & 6 & 4 & 12 & - & - & 4 & 9 & 4 & 5 & 6 & 10 & 4 & 6 & 8 & 10 & - & 6 & 7 & 8 & 128 \\
\hline Eristalis $\mathrm{sp}$. & - & 6 & - & 4 & - & 3 & 4 & 8 & 4 & - & 3 & 7 & - & 3 & 2 & 7 & 2 & - & - & 6 & 4 & 3 & - & 8 & 74 \\
\hline Palpada sp. & 2 & 3 & - & 3 & - & - & 4 & 3 & 3 & - & - & 5 & - & 4 & 3 & - & 2 & - & - & 3 & - & - & - & 4 & 39 \\
\hline Helophilus trivittatusWeid & 4 & 4 & 6 & 8 & 3 & 5 & - & 9 & - & 8 & 6 & 10 & 3 & 4 & 7 & 9 & 4 & 2 & - & 7 & 3 & 4 & 8 & 12 & 126 \\
\hline Helophilus sp. & 2 & - & 3 & 4 & 2 & - & - & 5 & 3 & - & 4 & 6 & - & - & 3 & 7 & - & - & - & 3 & - & - & 3 & 2 & 47 \\
\hline Syritta $\mathrm{sp}$. & 2 & - & - & 2 & - & - & 3 & 6 & - & - & - & 3 & 2 & 2 & - & 4 & - & - & - & 2 & - & - & - & - & 26 \\
\hline Sub total & 37 & 39 & 41 & 71 & 42 & 40 & 24 & 93 & 41 & 28 & 37 & 77 & 44 & 40 & 41 & 79 & 31 & 38 & 28 & 77 & 30 & 43 & 41 & 74 & 1136 \\
\hline \multicolumn{26}{|l|}{ Sub-Family Syrphinae } \\
\hline $\begin{array}{ll}\text { Epistrophe } \\
\text { (Meigen) }\end{array}$ & 4 & 3 & 2 & 7 & - & 6 & - & 8 & 3 & 7 & 3 & 9 & - & - & 4 & 6 & 3 & 2 & - & 4 & - & - & 4 & 6 & 81 \\
\hline $\begin{array}{l}\text { Episyrphusbalteatus } \\
\text { Geer) }\end{array}$ & 3 & 5 & 5 & 8 & - & 7 & 4 & 8 & - & 6 & - & 11 & - & 6 & 7 & 9 & - & 7 & - & 8 & - & 3 & 2 & 6 & 105 \\
\hline Eupeodu s corolla (Fabricius) & 8 & 6 & 5 & 14 & 5 & 6 & 6 & 15 & 7 & 2 & 6 & 5 & 4 & 2 & - & 13 & 8 & 4 & 6 & 3 & 6 & 4 & - & 13 & 148 \\
\hline Scaeva pyrastri (Linneaus) & 4 & - & 4 & 6 & - & - & 3 & 4 & 2 & 3 & - & 7 & - & 3 & 3 & 5 & - & - & 4 & 6 & 7 & - & 3 & 6 & 70 \\
\hline $\begin{array}{l}\text { Sphaerophoria bengalensis } \\
\text { Mac. }\end{array}$ & 6 & 4 & 5 & 11 & 3 & 4 & - & 8 & - & 4 & 5 & 7 & 4 & - & 5 & 7 & 6 & - & 4 & 3 & 9 & - & 3 & 14 & 112 \\
\hline Sphaerophoria indiana Bigot & 4 & - & 4 & 12 & 3 & 6 & - & 7 & 4 & 5 & 4 & 8 & - & 5 & - & 10 & 4 & - & - & 6 & - & 4 & 6 & 9 & 101 \\
\hline $\begin{array}{l}\begin{array}{l}\text { Sphaerophoria } \\
\text { (Linnaeus) }\end{array} \\
\end{array}$ & 5 & 7 & 4 & 14 & 3 & - & 5 & 8 & 10 & 4 & 4 & - & 8 & 3 & 6 & 7 & 12 & - & 4 & - & 8 & 4 & - & 15 & 131 \\
\hline Syrphus sp. & 2 & - & - & 4 & 5 & 3 & - & 6 & 2 & - & - & 8 & 4 & 3 & - & 4 & - & 3 & 2 & 4 & 5 & 2 & 3 & 6 & 66 \\
\hline Sub total & 36 & 25 & 29 & 76 & 19 & 32 & 18 & 64 & 28 & 31 & 22 & 55 & 20 & 22 & 25 & 61 & 33 & \begin{tabular}{|l|l}
16 \\
\end{tabular} & 20 & 34 & 35 & \begin{tabular}{|l|}
17 \\
\end{tabular} & 21 & 75 & 814 \\
\hline Total individuals & 73 & \begin{tabular}{|l|}
64 \\
\end{tabular} & 70 & 147 & 61 & \begin{tabular}{|l|}
72 \\
\end{tabular} & 42 & 157 & 69 & 59 & 59 & 132 & 64 & 62 & 66 & 140 & 64 & \begin{tabular}{|l|}
54 \\
\end{tabular} & 48 & 111 & 65 & \begin{tabular}{|l|}
60 \\
\end{tabular} & 62 & 149 & 1950 \\
\hline Total Species & 17 & 15 & 17 & 19 & 14 & 18 & 14 & 21 & 16 & \begin{tabular}{|l|l}
16 & \\
\end{tabular} & 16 & 19 & 14 & 16 & 15 & 19 & 14 & 13 & 14 & 18 & 12 & 14 & 15 & 18 & 20 \\
\hline
\end{tabular}


Table.3 Relative abundance of Syrphid flies in major flowers of Kashmir during 2013

\begin{tabular}{|c|c|c|c|c|c|c|c|c|c|c|c|c|c|c|c|c|c|c|c|c|c|c|c|c|c|c|c|}
\hline \multirow{4}{*}{ Sub-family, species } & \multicolumn{26}{|c|}{ Abundance of Syrphid flies in major flowers of Kashmir (\%) } & \multirow{4}{*}{$\begin{array}{l}\text { Total } \\
\text { mean }\end{array}$} \\
\hline & \multicolumn{13}{|c|}{ Srinagar } & \multicolumn{13}{|c|}{ Baramullah } & \\
\hline & \multicolumn{4}{|c|}{ Shalimar } & \multicolumn{4}{|c|}{ Harwan } & \multicolumn{4}{|c|}{ Gulabagh } & \multicolumn{5}{|c|}{ Wagoora } & \multicolumn{4}{|c|}{ Mamoosa } & \multicolumn{4}{|c|}{ Warpora } & \multirow[t]{2}{*}{ Mean } & \\
\hline & Daff. & Ro & \begin{tabular}{|l|} 
Chry \\
\end{tabular} & WFs & Daff. & Ro & Chry & WFs & Daff. & Ro & Chry & $\overline{W F s}$ & Mean & Daff. & Ro & Chry & WFs & Daff. & Ro & Chry & WFs & Daff. & Ro & Chry & WFs & & \\
\hline \multicolumn{28}{|l|}{$\begin{array}{l}\text { Sub-Family: } \\
\text { Eristalinae }\end{array}$} \\
\hline $\begin{array}{l}\text { Eristalinus taeniops } \\
\text { (Wied.) }\end{array}$ & 7.81 & $\overline{-}$ & - & 2.67 & 6.06 & 4.05 & 3.38 & 2.29 & 9.37 & - & - & 1.22 & 3.15 & 10.52 & 3.63 & 2.89 & - & 5.55 & - & - & 2.89 & - & - & 5.88 & 1.92 & 2.77 & 2.96 \\
\hline $\begin{array}{l}\text { Eristalinus paria Bigot } \\
\end{array}$ & \begin{tabular}{|l|}
4.68 \\
\end{tabular} & 2.40 & - & 2.67 & 4.54 & - & 3.38 & 1.64 & - & - & - & 2.45 & 1.81 & - & - & 4.34 & 2.73 & 8.33 & - & 4.34 & 2.89 & - & - & - & 3.20 & 2.15 & 1.98 \\
\hline $\begin{array}{l}\text { Eristalinus aeneus } \\
\text { (Scopoli) }\end{array}$ & 9.37 & 4.81 & 8.10 & 5.34 & 10.6 & 10.81 & 6.77 & 4.94 & 12.5 & 12.5 & \begin{tabular}{|l|}
5.66 \\
\end{tabular} & 4.90 & 8.02 & 5.26 & 9.09 & 4.34 & 5.47 & - & - & 10.86 & 7.24 & 9.67 & 7.14 & 11.76 & 6.41 & 7.10 & 7.56 \\
\hline $\begin{array}{l}\text { Eristalinus } s p . \\
\end{array}$ & 3.12 & 2.40 & - & 3.20 & 3.03 & 4.05 & 5.08 & 2.74 & - & - & - & 1.84 & 2.11 & - & - & - & 2.73 & 5.55 & - & - & 2.89 & - & - & - & - & 0.93 & 1.52 \\
\hline $\begin{array}{l}\text { Eristalis arbustorum } \\
(L .)\end{array}$ & 7.81 & 4.81 & 4.05 & 3.20 & 4.54 & 2.70 & - & 3.84 & - & - & 5.66 & 2.45 & 3.25 & - & 7.27 & - & 4.10 & 8.33 & 4.44 & - & 3.62 & 12.90 & - & 11.76 & 5.12 & 4.79 & 4.02 \\
\hline $\begin{array}{l}\begin{array}{l}\text { Eoseristalis cerealis } \\
\text { (Fabicius) }\end{array} \\
\end{array}$ & 12.5 & 8.43 & 8.10 & 8.02 & 9.09 & 12.16 & 8.47 & 8.24 & 28.12 & 12.5 & 9.43 & 7.36 & 11.03 & 18.42 & 7.27 & 7.24 & 7.53 & 16.66 & 11.11 & \begin{tabular}{|l|}
15.21 \\
\end{tabular} & 6.52 & - & 9.52 & 11.76 & 8.33 & 9.96 & 10.49 \\
\hline $\begin{array}{l}\text { Eristalis tenax } \\
\text { (Linnaeus) }\end{array}$ & 6.25 & \begin{tabular}{|l|}
10.84 \\
\end{tabular} & \begin{tabular}{|l|l|}
10.81 &
\end{tabular} & 8.02 & 7.57 & 9.45 & \begin{tabular}{|l|}
11.86 \\
\end{tabular} & \begin{tabular}{|l|l|}
6.59 \\
\end{tabular} & - & 18.75 & \begin{tabular}{|l|}
15.0 \\
\end{tabular} & 8.58 & \begin{tabular}{|l|}
.47 \\
\end{tabular} & 21.0 & 16.36 & 8.69 & 9.58 & 8.33 & 20.0 & \begin{tabular}{|l|}
17.39 \\
\end{tabular} & 9.42 & 12.90 & 19.0 & 11.76 & 9.61 & \begin{tabular}{|l|}
13.67 \\
\end{tabular} & 11.57 \\
\hline $\begin{array}{l}\text { Eristalis interruptus } \\
\text { (Poda) }\end{array}$ & 4.68 & 6.02 & \begin{tabular}{|l|l|}
9.45 \\
\end{tabular} & 8.02 & 3.03 & 6.75 & 5.08 & \begin{tabular}{|l|l|}
8.79 \\
\end{tabular} & - & - & 3.77 & 7.97 & \begin{tabular}{|l|}
5.29 \\
\end{tabular} & - & \begin{tabular}{|l|}
14.54 \\
\end{tabular} & 10.14 & 7.53 & - & 11.11 & \begin{tabular}{|l|}
19.56 \\
\end{tabular} & 10.14 & 6.45 & 9.52 & 13.72 & 8.33 & 9.25 & 7.27 \\
\hline Eristalis $\mathrm{sp}$. & \begin{tabular}{|l|}
3.12 \\
\end{tabular} & 4.81 & - & 3.20 & 4.54 & - & - & \begin{tabular}{|l|}
4.94 \\
\end{tabular} & 6.25 & 4.16 & - & 3.06 & 2.84 & - & - & \begin{tabular}{|l|l|}
4.34 \\
\end{tabular} & 4.10 & - & 6.66 & 4.34 & 3.62 & 6.45 & 7.14 & - & 5.76 & 3.53 & 3.18 \\
\hline Palpada sp. & - & - & \begin{tabular}{|l|}
2.70 \\
\end{tabular} & 2.13 & 3.03 & 2.70 & - & \begin{tabular}{|l|}
2.74 \\
\end{tabular} & - & - & - & 2.45 & 1.31 & 5.26 & - & 2.89 & - & - & - & - & 3.62 & 6.45 & - & - & 1.92 & 1.67 & 1.49 \\
\hline $\begin{array}{l}\begin{array}{l}\text { Helophilus } \\
\text { trivittatusWeid }\end{array} \\
\end{array}$ & \begin{tabular}{|l|}
4.68 \\
\end{tabular} & 7.22 & \begin{tabular}{|l|l|}
6.75 \\
\end{tabular} & $\begin{array}{l}5.34 \\
\end{array}$ & 6.06 & 8.10 & 5.08 & \begin{tabular}{|l|}
4.39 \\
\end{tabular} & 6.25 & \begin{tabular}{|l|}
14.58 \\
\end{tabular} & 9.43 & 6.74 & \begin{tabular}{|l|}
7.05 \\
\end{tabular} & - & 9.09 & 8.69 & 5.47 & 8.33 & - & - & 4.34 & \begin{tabular}{|l|}
16.12 \\
\end{tabular} & \begin{tabular}{|l|}
16.66 \\
\end{tabular} & 5.88 & 7.69 & 6.85 & 6.95 \\
\hline Helophilus sp. & - & - & - & 2.67 & - & 2.70 & 5.08 & \begin{tabular}{|l|}
3.29 \\
\end{tabular} & - & - & 5.66 & 3.68 & 1.92 & - & - & - & 3.42 & - & - & 4.34 & 3.62 & - & - & - & 3.84 & 1.26 & 1.59 \\
\hline Syritta sp. & - & - & - & 2.67 & - & 2.70 & - & \begin{tabular}{|l|}
3.29 \\
\end{tabular} & - & - & 3.77 & 2.45 & 1.24 & - & - & - & 2.73 & - & - & - & 2.17 & - & - & - & - & 0.40 & 0.82 \\
\hline Sub total & 64.06 & \begin{tabular}{|l|}
51.81 \\
\end{tabular} & \begin{tabular}{|l|}
50.00 \\
\end{tabular} & \begin{tabular}{|l|}
57.22 \\
\end{tabular} & 62.12 & 66.22 & \begin{tabular}{|l|l|}
54.37 \\
\end{tabular} & \begin{tabular}{|l|}
58.79 \\
\end{tabular} & 62.50 & \begin{tabular}{|l|}
62.50 \\
\end{tabular} & \begin{tabular}{|l|l|}
58.49 \\
\end{tabular} & 55.25 & \begin{tabular}{|l|}
58.49 \\
\end{tabular} & 60.56 & 67.29 & 53.56 & \begin{tabular}{|l|}
55.54 \\
\end{tabular} & \begin{tabular}{|l|}
61.14 \\
\end{tabular} & \begin{tabular}{|l|l|}
53.37 \\
\end{tabular} & \begin{tabular}{|l|}
76.38 \\
\end{tabular} & 63.04 & 70.94 & \begin{tabular}{|l|}
69.05 \\
\end{tabular} & 72.52 & 62.22 & \begin{tabular}{|l|l|}
64.37 \\
\end{tabular} & 61.40 \\
\hline \multicolumn{28}{|l|}{$\begin{array}{l}\text { Sub- } \\
\text { Family:Syrphinae }\end{array}$} \\
\hline $\begin{array}{l}\begin{array}{l}\text { Epistrophe } \\
\text { grossulariae (Meigen) }\end{array} \\
\end{array}$ & 4.68 & 6.02 & \begin{tabular}{|l|l|}
5.40 \\
\end{tabular} & 4.27 & 3.03 & 5.40 & 3.38 & \begin{tabular}{|l|l|}
3.84 \\
\end{tabular} & - & \begin{tabular}{|l|}
10.41 \\
\end{tabular} & 7.54 & 4.29 & \begin{tabular}{|l|l|}
4.85 \\
\end{tabular} & 7.89 & 3.63 & 2.89 & 3.42 & - & - & 4.34 & 3.62 & 6.45 & - & 3.92 & 2.56 & 3.22 & 4.03 \\
\hline $\begin{array}{l}\text { Episyrphusbalteatus(De } \\
\text { Geer) }\end{array}$ & \begin{tabular}{|l|l|}
4.68 \\
\end{tabular} & 7.22 & 6.75 & 5.34 & 3.03 & 6.75 & 6.77 & \begin{tabular}{|l|l|}
4.94 &
\end{tabular} & - & 6.25 & 3.77 & 7.97 & \begin{tabular}{|l|}
5.28 \\
\end{tabular} & - & 9.09 & 7.24 & 5.47 & 5.55 & \begin{tabular}{|l|l|}
11.11 \\
\end{tabular} & - & 7.24 & - & - & 3.92 & 2.56 & 4.34 & 4.81 \\
\hline $\begin{array}{l}\text { Eupeodus corolla } \\
\text { (Fabricius) }\end{array}$ & 10.93 & \begin{tabular}{|l|}
10.84 \\
\end{tabular} & \begin{tabular}{|l|l|}
10.81 & \\
\end{tabular} & 6.41 & 9.09 & 9.45 & \begin{tabular}{|l|}
13.55 \\
\end{tabular} & \begin{tabular}{|l|}
8.24 \\
\end{tabular} & 9.37 & 8.33 & \begin{tabular}{|l|l|}
3.77 & \\
\end{tabular} & 7.97 & \begin{tabular}{|l|}
9.06 \\
\end{tabular} & 13.15 & - & \begin{tabular}{|l|l|}
10.14 \\
\end{tabular} & 9.58 & \begin{tabular}{|l|}
13.88 \\
\end{tabular} & \begin{tabular}{|l|l|}
8.88 &
\end{tabular} & 6.52 & 5.07 & \begin{tabular}{|l|}
16.12 \\
\end{tabular} & 16.66 & 3.92 & 6.41 & 9.19 & 9.12 \\
\hline $\begin{array}{l}\begin{array}{l}\text { Scaeva pyrastri } \\
\text { (Linneaus) }\end{array} \\
\text { (L) }\end{array}$ & - & 4.81 & \begin{tabular}{|l|}
4.05 \\
\end{tabular} & 3.74 & 3.03 & - & - & \begin{tabular}{|l|}
3.29 \\
\end{tabular} & 9.37 & - & - & 3.06 & \begin{tabular}{|l|}
2.61 \\
\end{tabular} & - & - & 4.34 & 4.10 & 5.55 & 4.44 & - & 3.62 & - & - & - & 3.84 & 2.15 & 2.38 \\
\hline \begin{tabular}{|l}
$\begin{array}{l}\text { Sphaerophoria } \\
\text { bengalensis Mac. }\end{array}$ \\
\end{tabular} & 7.81 & 3.61 & \begin{tabular}{|l|}
5.40 \\
\end{tabular} & 6.41 & 4.54 & 2.70 & 3.38 & \begin{tabular}{|l|l|}
5.49 &
\end{tabular} & 6.25 & 4.16 & - & 6.13 & \begin{tabular}{|l|}
4.65 \\
\end{tabular} & 7.89 & 7.27 & 4.34 & 6.16 & - & \begin{tabular}{|l|l}
6.66 \\
\end{tabular} & 8.69 & 5.07 & - & 7.14 & 3.92 & 8.33 & 5.45 & 5.05 \\
\hline $\begin{array}{l}\text { Sphaerophoria indiana } \\
\text { Bigot }\end{array}$ & - & 4.01 & \begin{tabular}{|l|}
6.75 \\
\end{tabular} & 6.41 & 4.54 & 2.70 & \begin{tabular}{|l|}
10.16 \\
\end{tabular} & 7.14 & 12.5 & - & 5.66 & 4.90 & \begin{tabular}{|l|}
5.39 \\
\end{tabular} & - & 7.27 & \begin{tabular}{|l|l|}
2.89 &
\end{tabular} & 5.47 & - & \begin{tabular}{|l|l}
4.44 &
\end{tabular} & - & \begin{tabular}{|l|}
2.89 \\
\end{tabular} & - & 7.14 & 3.92 & 5.76 & 3.31 & 4.35 \\
\hline $\begin{array}{l}\text { Sphaerophoria scripta } \\
\text { (Linnaeus) }\end{array}$ & 7.81 & 7.22 & \begin{tabular}{|l|}
6.75 \\
\end{tabular} & 6.95 & 6.06 & 4.05 & 8.47 & 5.49 & - & 8.33 & 16.98 & 7.36 & \begin{tabular}{|l|}
7.12 \\
\end{tabular} & 10.52 & 5.45 & \begin{tabular}{|l|}
11.59 \\
\end{tabular} & 7.53 & 5.55 & \begin{tabular}{|l|l|}
6.66 \\
\end{tabular} & 4.34 & \begin{tabular}{|l|}
3.62 \\
\end{tabular} & - & - & 7.84 & 5.76 & 5.73 & 6.42 \\
\hline Syrphus sp. & - & 3.61 & \begin{tabular}{|l|}
4.05 \\
\end{tabular} & 3.20 & 4.54 & 2.70 & - & 2.74 & - & - & 3.77 & 3.06 & \begin{tabular}{|l|}
2.30 \\
\end{tabular} & - & - & \begin{tabular}{|l|}
2.89 \\
\end{tabular} & 2.73 & 8.33 & 4.44 & - & \begin{tabular}{|l|}
4.34 \\
\end{tabular} & 6.45 & - & - & 2.56 & \begin{tabular}{|l|}
2.64 \\
\end{tabular} & 2.47 \\
\hline Sub total & 35.94 & \begin{tabular}{|l|}
48.19 \\
\end{tabular} & \begin{tabular}{|l|}
50.00 \\
\end{tabular} & 42.78 & 37.88 & 33.78 & \begin{tabular}{|l|}
45.63 \\
\end{tabular} & 41.21 & 37.50 & \begin{tabular}{|l|}
37.50 \\
\end{tabular} & 41.51 & \begin{tabular}{|l|l|}
44.75 \\
\end{tabular} & 41.51 & 39.45 & \begin{tabular}{|l|}
32.71 \\
\end{tabular} & \begin{tabular}{|l|}
46.42 \\
\end{tabular} & \begin{tabular}{|l|l|}
44.46 \\
\end{tabular} & \begin{tabular}{|l|}
38.86 \\
\end{tabular} & 46.63 & \begin{tabular}{|l|}
23.62 \\
\end{tabular} & \begin{tabular}{|l|}
36.96 \\
\end{tabular} & 29.06 & 30.95 & 27.48 & 37.78 & 36.03 & 38.60 \\
\hline Total individuals & 64 & 83 & 74 & 187 & 66 & 74 & 59 & 182 & 32 & 48 & 53 & 163 & 1085 & 38 & 55 & 69 & 146 & 36 & 45 & 46 & 138 & 31 & 42 & 51 & 156 & 853 & 1938 \\
\hline Total species & 15 & 17 & 15 & 21 & 19 & 18 & 15 & 21 & 9 & 10 & 14 & 21 & & 9 & 12 & 17 & 19 & 12 & 12 & 11 & 21 & 10 & 9 & 13 & 19 & & 21 \\
\hline
\end{tabular}


Table.4 Relative abundance of Syrphid flies in major flowers of Kashmir during 2014

\begin{tabular}{|c|c|c|c|c|c|c|c|c|c|c|c|c|c|c|c|c|c|c|c|c|c|c|c|c|c|c|c|}
\hline \multirow[t]{4}{*}{ Sub-family, species } & \multicolumn{26}{|c|}{ Abundance of Syrphid flies in major flowers of Kashmir (\%) } & \multirow{4}{*}{ Total } \\
\hline & \multicolumn{12}{|c|}{ Srinagar } & \multirow{3}{*}{ Mean } & \multicolumn{12}{|c|}{ Baramullah } & \multirow{3}{*}{ Mean } & \\
\hline & \multicolumn{4}{|c|}{ Shalimar } & \multicolumn{4}{|c|}{ Harwan } & \multicolumn{4}{|c|}{ Gulabagh } & & \multicolumn{4}{|c|}{ Wagoora } & \multicolumn{4}{|c|}{ Mamoosa } & \multicolumn{4}{|c|}{ Warpora } & & \\
\hline & Daff. & Ro & Chry & WFs & Daff. & Ro & Chry & WFs & Daff. & Ro & Chry & WFs & & Daff. & Ro & Chry & WFs & Daff. & Ro & Chry & WFs & Daff. & Ro & Chry & WFs & & \\
\hline \multicolumn{28}{|l|}{ Sub-Family: Eristalinae } \\
\hline $\begin{array}{l}\begin{array}{l}\text { Eristalinus } \\
\text { (Wiedemann) }\end{array} \\
\end{array}$ & - & 6.25 & 2.85 & 2.04 & 8.19 & 2.77 & - & 1.91 & 4.34 & 3.38 & - & - & 2.64 & 4.68 & - & - & 2.14 & 9.37 & 7.40 & 6.25 & - & 3.07 & 3.33 & - & 1.34 & 3.13 & 3.00 \\
\hline Eristalinus paria Bigot & 2.73 & 3.12 & 4.28 & - & 6.55 & & - & 1.91 & 7.24 & 6.77 & - & 1.51 & 2.84 & 6.25 & - & 4.54 & 1.42 & 4.68 & 3.70 & - & - & 6.15 & - & 3.22 & - & 2.49 & 2.67 \\
\hline $\begin{array}{l}\begin{array}{l}\text { Eristalinus } \\
\text { (Scopoli) }\end{array} \\
\end{array}$ & 5.47 & 4.68 & 5.71 & 5.44 & 9.83 & 6.94 & 7.14 & 7.64 & 4.34 & 6.77 & 5.08 & 6.06 & 6.25 & 10.93 & 4.83 & 6.06 & 7.14 & 4.68 & 7.40 & 6.25 & 10.81 & - & 10.0 & 4.83 & 6.04 & 6.58 & 6.41 \\
\hline Eristalinus $s p$. & - & - & \begin{tabular}{|l|}
2.85 \\
\end{tabular} & - & \begin{tabular}{|l|l|}
6.55 \\
\end{tabular} & 4.16 & - & 1.27 & $\begin{array}{ll}4.34 \\
\end{array}$ & - & 6.77 & 1.51 & 2.28 & 3.12 & 3.22 & - & 1.42 & \begin{tabular}{|l|}
3.12 \\
\end{tabular} & 3.70 & 8.33 & 1.80 & - & \begin{tabular}{|l|}
6.66 \\
\end{tabular} & 4.83 & - & \begin{tabular}{|l|}
3.01 \\
\end{tabular} & 2.64 \\
\hline $\begin{array}{l}\begin{array}{l}\text { Eristalis arbustorum } \\
\text { (L.) }\end{array} \\
\end{array}$ & 8.21 & 4.68 & 5.71 & 2.04 & 6.55 & 4.16 & 4.76 & 3.18 & 4.34 & 3.38 & - & 1.51 & 4.04 & 6.25 & 3.22 & 4.54 & - & - & 7.40 & - & 4.50 & 3.07 & 6.66 & 4.83 & 2.68 & 3.60 & 3.82 \\
\hline $\begin{array}{l}\begin{array}{l}\text { Eoseristalis } \\
\text { (Fabicius) }\end{array} \\
\end{array}$ & 12.32 & 7.81 & 5.71 & 8.84 & 11.47 & 8.33 & - & 9.55 & 11.59 & - & 13.55 & 7.57 & 8.06 & 12.5 & 14.51 & 7.57 & 8.57 & - & 14.81 & 12.5 & 12.61 & 10.76 & 8.33 & 9.67 & 7.38 & 9.93 & 9.00 \\
\hline $\begin{array}{ll}\begin{array}{l}\text { Eristalis } \\
\text { (Linnaeus) }\end{array} & \text { tenax } \\
\end{array}$ & 8.21 & 6.25 & \begin{tabular}{|l|}
10.0 \\
\end{tabular} & 10.20 & \begin{tabular}{|l|l|}
11.47 \\
\end{tabular} & 9.72 & 9.52 & 6.36 & 8.69 & 13.55 & 8.47 & 9.84 & 9.35 & 10.93 & 9.67 & 7.57 & 9.28 & 7.81 & 11.11 & \begin{tabular}{|l|}
8.33 \\
\end{tabular} & 11.71 & 12.30 & 15.0 & 9.67 & 9.39 & \begin{tabular}{|l|}
10.23 \\
\end{tabular} & 9.80 \\
\hline $\begin{array}{l}\begin{array}{l}\text { Eristalis } \\
\text { (Poda) }\end{array} \\
\end{array}$ & & 7.81 & 8.57 & 5.44 & - & 8.33 & 9.52 & 7.64 & - & - & 6.77 & 6.81 & 5.07 & 6.25 & 8.06 & 9.09 & 7.14 & 6.25 & 11.11 & \begin{tabular}{|l|l|}
16.66 \\
\end{tabular} & 9.0 & - & 10.0 & 11.29 & 5.36 & \begin{tabular}{|l|}
8.35 \\
\end{tabular} & 6.71 \\
\hline Eristalis sp. & - & 9.37 & - & 2.72 & - & 4.16 & 9.52 & 5.09 & 5.79 & - & 5.08 & 5.30 & 3.91 & - & 4.83 & 3.03 & 5.0 & \begin{tabular}{|l|}
3.12 \\
\end{tabular} & - & - & 5.40 & 6.15 & 5.0 & - & 5.36 & 3.15 & 3.53 \\
\hline Palpada sp. & 2.73 & 4.68 & - & 2.04 & - & - & 9.52 & 1.91 & 4.34 & - & - & 3.78 & 2.42 & - & 6.45 & 4.54 & - & \begin{tabular}{|l|}
3.12 \\
\end{tabular} & - & - & 2.70 & - & - & - & 2.68 & 1.62 & 2.02 \\
\hline $\begin{array}{l}\text { Helophilus } \\
\text { trivittatusWeid }\end{array}$ & 5.47 & 6.25 & 8.57 & 5.44 & \begin{tabular}{|l}
4.91 \\
\end{tabular} & 6.94 & - & 5.73 & - & 13.55 & 10.16 & 7.57 & 6.21 & 4.68 & 6.45 & 10.60 & 6.42 & 6.25 & 3.70 & - & 6.30 & 4.61 & 6.66 & 12.90 & 8.05 & 6.38 & 6.30 \\
\hline Helophilus sp. & 2.73 & - & 4.28 & 2.72 & \begin{tabular}{|l|l|}
3.27 \\
\end{tabular} & 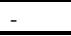 & - & 3.18 & 4.34 & - & 6.77 & 4.54 & 2.65 & - & - & 4.54 & 5.0 & - & - & - & 2.70 & - & - & 4.83 & 1.34 & 1.53 & 2.10 \\
\hline Syritta sp. & 2.73 & - & - & 1.36 & - & - & 7.14 & 3.82 & - & - & - & 2.27 & 1.44 & 3.12 & \begin{tabular}{|l|}
3.22 \\
\end{tabular} & - & 2.85 & - & - & - & 1.80 & - & - & - & - & 0.91 & 1.17 \\
\hline Sub total & 50.68 & 60.93 & 58.53 & 48.30 & 68.79 & 55.55 & 57.12 & 59.23 & 59.42 & 47.46 & 62.75 & 58.33 & 57.33 & 68.71 & \begin{tabular}{|l|}
64.56 \\
\end{tabular} & 62.14 & 56.48 & 48.45 & 70.33 & \begin{tabular}{|l|l|}
58.34 \\
\end{tabular} & 69.33 & 46.18 & 71.69 & 66.17 & 49.61 & 61.01 & 58.26 \\
\hline \multicolumn{28}{|l|}{ Sub-Family Syrphinae } \\
\hline $\begin{array}{l}\begin{array}{l}\text { Epistrophe } \\
\text { grossulariae (Meigen) }\end{array} \\
\end{array}$ & 5.47 & 4.68 & 2.85 & 4.76 & - & 8.33 & - & 5.09 & 4.34 & 11.86 & 5.08 & 6.81 & 4.93 & - & - & 6.06 & 4.28 & 4.68 & 3.70 & - & 3.60 & - & - & 6.45 & 4.02 & 2.73 & 3.83 \\
\hline $\begin{array}{l}\text { Episyrphusbalteatus } \\
\text { (De Geer) }\end{array}$ & 4.10 & 7.81 & 7.14 & 5.44 & - & 9.72 & 9.52 & 5.09 & - & 10.16 & - & 8.33 & 5.61 & - & 9.67 & 10.60 & 6.42 & - & 12.96 & - & 7.20 & - & 5.0 & 3.22 & 4.02 & 4.92 & 5.30 \\
\hline $\begin{array}{lll}\begin{array}{l}\text { Eupeodu } \\
\text { (Fabricius) }\end{array} & \text { corolla } \\
\end{array}$ & 10.95 & 9.37 & 7.14 & 9.52 & 8.19 & 8.33 & 14.28 & 9.55 & 10.14 & 3.38 & 10.16 & 3.78 & 8.73 & 6.25 & 3.22 & - & 9.28 & 12.5 & 7.40 & 12.5 & 2.70 & 9.23 & 6.66 & - & 8.72 & 6.53 & 7.63 \\
\hline $\begin{array}{ll}\begin{array}{l}\text { Scaeva } \\
\text { (Linneaus) }\end{array} & \text { pyrastri } \\
\end{array}$ & 5.47 & - & 5.71 & 4.08 & - & 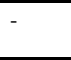 & 7.14 & 2.54 & 2.89 & 5.08 & - & 5.30 & 3.18 & - & 4.83 & 4.54 & 3.57 & - & - & 8.33 & 5.40 & 10.76 & - & 4.83 & 4.02 & 3.85 & 3.52 \\
\hline $\begin{array}{l}\text { Sphaerophoria } \\
\text { bengalensis Macqaurt }\end{array}$ & 8.21 & 6.25 & 7.14 & 7.48 & 4.91 & 5.55 & - & 5.09 & - & 6.77 & 8.47 & 5.30 & 5.43 & 6.25 & - & 7.57 & 5.0 & 9.37 & - & 8.33 & 2.70 & 13.84 & - & 4.83 & 9.39 & 5.60 & 5.52 \\
\hline $\begin{array}{l}\text { Sphaerophoria indiana } \\
\text { Bigot }\end{array}$ & 5.47 & - & 5.71 & 8.16 & 4.91 & 8.33 & - & 4.45 & 5.79 & 8.47 & 6.77 & 6.06 & 5.34 & - & 8.06 & - & 7.14 & 6.25 & - & - & 5.40 & - & 6.66 & 9.67 & 6.04 & 4.10 & 4.72 \\
\hline $\begin{array}{l}\text { Sphaerophoria scripta } \\
\text { (Linnaeus) }\end{array}$ & 6.84 & 10.93 & 5.71 & 9.52 & 4.91 & - & 11.90 & 5.09 & 14.49 & 6.77 & 6.77 & 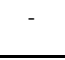 & 6.91 & 12.5 & 4.83 & 9.09 & 5.0 & 18.75 & - & 8.33 & & 12.30 & 6.66 & - & 10.06 & 7.30 & 7.10 \\
\hline Syrphus sp. & 2.73 & - & - & 2.72 & \begin{tabular}{|l|l|}
8.19 \\
\end{tabular} & 4.16 & - & 3.82 & 2.89 & & - & 6.06 & 2.54 & 6.25 & \begin{tabular}{|l}
4.83 \\
\end{tabular} & - & 2.85 & - & 5.55 & 4.16 & 3.60 & 7.69 & 3.33 & 4.83 & 4.02 & 3.92 & 3.23 \\
\hline Sub total & 49.32 & 39.05 & 41.47 & 51.70 & 31.21 & 44.45 & 42.84 & 40.73 & 40.58 & 52.54 & 37.25 & 41.67 & 42.67 & 31.29 & 35.44 & 37.86 & 43.52 & 51.55 & 29.66 & \begin{tabular}{|l|l|}
41.66 \\
\end{tabular} & 30.66 & 53.82 & 28.31 & 33.83 & 50.29 & \begin{tabular}{|l|}
38.99 \\
\end{tabular} & 41.74 \\
\hline Total individuals & 73 & 64 & 70 & 147 & 61 & 72 & 42 & 157 & 69 & 59 & 59 & 132 & 1005 & 64 & 62 & 66 & 140 & 64 & 54 & 48 & 111 & 65 & 60 & 62 & 149 & 945 & 1950 \\
\hline Total Species & 17 & 15 & 17 & 19 & 14 & 18 & 14 & 21 & 16 & 16 & 16 & 19 & & 14 & 16 & 15 & 19 & 14 & 13 & 14 & 18 & 12 & 14 & 15 & 18 & & 20 \\
\hline
\end{tabular}

Daff = Daffodil, Ro= Rose, Chry = Chrysanthemum, WFs= Wild flowers (including Dandelion, Fennel and Black Berry), Number of samples= 120 sweeps in

each flower crops of each locations, Sampling method=sweep net method, Sampling time=April to September at weekly interval 
Int.J.Curr.Microbiol.App.Sci (2017) 6(9): 1539-1552

Table.5 Parameter of abundance of Syrphid flies in major flowers of Kashmir (2013-2014)

\begin{tabular}{|c|c|c|c|c|c|c|c|c|c|c|c|}
\hline \multirow[t]{2}{*}{ Districts } & \multirow[t]{2}{*}{ Sites } & \multicolumn{2}{|c|}{$\mathrm{S}$} & \multicolumn{2}{|c|}{$\mathrm{N}$} & \multicolumn{2}{|c|}{ Diversity } & \multicolumn{2}{|c|}{ Richness } & \multirow{2}{*}{$\begin{array}{c}\text { Mean } \\
\text { Diversity } \\
\text { (H) }\end{array}$} & \multirow{2}{*}{$\begin{array}{c}\text { Mean } \\
\text { Richness } \\
\text { (Da) }\end{array}$} \\
\hline & & 2013 & 2014 & 2013 & 2014 & 2013 & 2014 & 2013 & 2014 & & \\
\hline \multirow[t]{3}{*}{ Srinagar } & Shalimar & 21 & 21 & 408 & 254 & 2.818 & 2.843 & 3.327 & 3.611 & 2.830 & 3.469 \\
\hline & Harwan & 21 & 21 & 381 & 332 & 2.900 & 2.970 & 3.365 & 3.445 & 2.935 & 3.405 \\
\hline & Gulabagh & 21 & 21 & 296 & 319 & 2.827 & 2.894 & 3.214 & 3.469 & 2.860 & 3.341 \\
\hline \multirow[t]{3}{*}{ Baramullah } & Wagoora & 21 & 21 & 308 & 332 & 2.835 & 2.895 & 3.490 & 3.445 & 2.865 & 3.467 \\
\hline & Mamoosa & 21 & 21 & 265 & 277 & 2.820 & 2.841 & 3.584 & 3.556 & 2.830 & 3.570 \\
\hline & Warpora & 21 & 21 & 280 & 308 & 2.738 & 2.754 & 3.549 & 3.490 & 2.746 & 3.519 \\
\hline
\end{tabular}


On the other hand, studies have shown that the smaller, sparsely hairy Syrphidae species (especially of the genera Melanostoma and Platycheirus) that visit reputedly anemophilous plants are relatively constant to at most two families of plants (Goot and Grabandt, 1970; Holloway, 1976; Leereveld, 1982). In present study, Eristalis tenax was found most abundant syrphid species followed by Eoseristalis cerealis and Eupeodus corolla while as Syritta sp. followed by Palpada sp. were least abundant species of syrphid fly in floricultural ecosystem of Kashmir which was similar to Khan and Riyaz (2017) findings and was reported that the Eristalis tenax followed by Eoseristalis cerealis, Eristalis interruptus and Episyrphus balteatus were pre-dominant species of syrphid flies in fruit orchards of Kashmir. Other authors like Mitra et al., (2005) also reported the quite similar abundance syrphid flies.

\section{Species diversity and richness of syrphid flies}

The parameters of abundance of syrphid flies in floricultural ecosystem of Kashmir during 2013 and 2014 are shown in table 5 and the parameters included species diversity and species richness. The species diversity was measured by the using Shannon-Wiener biodiversity index $(\mathrm{H})$ and species richness by using Margalef's richness index (Dmg). The parameters were calculated from two districts with three respective locations viz., Srinagar (Shalimar, Harwan and Gulabagh) and Baramullah (Wagoora, Mamoosa and Warpora) in floricultural ecosystem of Kashmir during 2013 and 2014.Data depicted that the highest species diversity $(\mathrm{H})$ of syrphid flies with 2.900 and 2.970 were recorded in the Harwan location of district Srinagar during 2013 and 2014 respectively while as least species diversity $(\mathrm{H})$ with 2.738 and 2.754 observed in the Warpora of district
Baramullah during 2013and 2014 respectively. The highest mean species diversity $\left(\mathrm{H}^{\prime}\right)$ of syrphid flies was found in Harwan of district Srinagar with 2.935 of floricultural ecosystem of Kashmir during the year 2013 and 2014 (Table 5).

The highest Species richness (Da) was observed in Mamoosa of district Baramullah with 3.584 and 3.611 in Shalimar of district Srinagar during 2013 and 2014 respectively. The least species richness (Da) was calculated as 3.214 in Gulabagh area of Srinagar district during the year 2013. In 2014, least species richness (Dmg!) was calculated as 3.445 in Harwan and Wagoora areas of district Srinagar and Baramullah respectively. The highest mean species richness of syrphid flies were observed as 3.570 in Mamoosa area of district Baramullah of floricultural ecosystem of Kashmir during the year 2013 and 2014 (Table 5). According to Shannon-wiener's diversity index (H') the calculated values showed that there is a big difference in the diversity of area which means the syrphid flies are not well scattered in all chosen locations of two districts of Kashmir due to varying topography, type of orchards and availability of aphids (Arif et al., 2014; Khan and Riyaz, 2017). The yielded values of Margalaf's richness index (Dmg) shown that richness of syrphid flies in the study area is not significantly different except Warpora location. Species diversity was low in areas with intensive human activity, and those species that occurred in these areas were generalists with broad ranges and good colonizing abilities. In contrast, undisturbed areas had more species and a higher representation of specialists (Bankowska, 1981; Ssymank, 2002).

The extensive study on biodiversity of aphidophagous syrphid flies were made in agro ecosystem of Kashmir during 2013 to 2014 and a total of 21 species of syrphid files 
were recorded from floriculture ecosystem of Kashmir. Among all species, Eristalis tenax was the most distributed and abundant species followed by Eoseristalis cerealis, Eupeodus corolla and Eristalis interruptus were recorded during both years while as Syritta $s p$. followed by Palpada sp. were least abundant species of syrphid fly in floricultural ecosystem of Kashmir. The highest mean species diversity $\left(\mathrm{H}^{!}\right)$of syrphid flies was found in Harwan of district Srinagar with 2.935 of floricultural ecosystem of Kashmir during the year 2013 and 2014. The highest mean species richness of syrphid flies were observed as 3.570 in Mamoosa area of district Baramullah of floricultural ecosystem of Kashmir during the year 2013 and 2014.

\section{Acknowledgements}

This research was funded by University Grant Commission (UGC), New Delhi, India entitled "Biodiversity and utilization of aphidophagous syrphid fly in agro-ecosystem of Kashmir". The author is thankful to UGC for the financial assistance.

\section{References}

Ankersmit, G.W., H. Dijkman, N.J. Keuning, H. Mertens, A. Sins and H. Tacoma. 1986. Episyrphus balteatus as a predator of the aphid Sitobion avenae on winter wheat. Entomol. Exp. Appl., 42: 271-277.

Arif, S., Khan, M.R., Shehzad, A., Nazir, N., Rahim, J., Naz, F., Rafi, R. 2014. Biodiversity of syrphid flies (Syrphidae: Diptera) from Poonch District of Azad Kashmir, Pakistan. J. Agril. Technol., 10(6):1465-1472.

Bankowska, R., 1981. Hover flies (Diptera, Syrphidae) of Warsaw and Mazovia, Memorabilia Zoologica 35: 57-78.

Colley, M.R., Luna J.M. 2000. Relative attractiveness of potential beneficial insectary plants to aphidophagous hoverfies (Diptera: Syrphidae). Environ. Entomol, 29: 1054-1059.

Cowgill, S.E., Wratten, S.D., Sotherton, N.W. 1993. The selective use of floral resources by the hoverfly Episyrphus balteatus (Diptera, Syrphidae) on farmland. Ann. Appl. Biol, 122:223-231.

Faegri, K., Van Der Pijl L. 1979. The principles of pollination ecology, Pergamon Oxford; 3rd ed., 244.

Ghahari, H., Hayat, R., Tabari M., Ostovan, H. 2008. Hoverflies (Diptera: Syrphidae) from rice fields and around grasslands of Northern Iran. Mun. Ent. Zool., 3(1): 275-283.

Ghorpade, K., Pathania, P.C. 2014. Hoverflies (Diptera-Syrphidae) in the collection of the Punjab Agricultural University, Ludhiana, India and a Checklist of those known from the Punjab Doab biogeographical sub-area. Colemania, 42:1-10.

Gilbert, F.S., 1981. Foraging ecology of hoverflies: morphology of the mouthparts in relation to feeding on nectar and pollen in some common urban species. Ecol. Entomol., 6:245262.

Goot, V.S., Grabandt. R.A.J. 1970. Some species of the genera Melanostoma, Platycheirus and Pyrophaena (Diptera: Syrphidae) and their relation to flowers Entomologische Berichten 30:135-143.

Haslett, J.R., 1989. Interpreting patterns of resource utilization: randomness and selectivity in pollen feeding by adult hoverflies. Oecologia, 78:433-442.

Heimpel, G.E., Jervis, M.A. 2005. Does floral nectar improve biological control by parasitoids? In: Plant-provided food for carnivorous insects: protective mutualism and its applications. $\mathrm{F}$. (Eds.): Wa"ckers P. van Rijn and J. Bruin. Cambridge University Press, Cambridge, United Kingdom. 
Holloway, B.A., 1976. Pollen-feeding in hover-flies (Diptera: Syrphidae). New Zealand J. Zoology, 3:339-350.

Jauker, F., Wolters, V. 2008. Hover flies are efficient pollinators of oilseed rape. Oecologia, 156:819-823.

Jervis, M.A., Kidd, N.A.C. 1996. Phytophagy. In: Insect Natural Enemies: Practical Approaches to their Study and Evaluation. (Eds.): M. Jervis and N. Kidd. Chapman and Hall, London, pp. 375-394.

Kearns, C. A., 2002. Flies and flowers: an enduring partnership. Wings (The Xerces Society) 25(2):3-8.

Khan, A. A., 2017b. Effect of insecticides on biodiversity of aphidophagous syrphid flies in fruit ecosystem of Kashmir. $J$. Entomol. Zool. Stud, 5(4):117-125.

Khan, A.A., 2017a. Functional Response of Four Syrphid Predators Associated with Mealy Cabbage Aphid, Brevicoryne brassicae L. on Cruciferous Vegetables. Int. J. Curr. Microbiol. App. Sci., 6(7):2806-2816.

Khan, A.A., Riyaz, S. 2017. Diversity and Distribution of Syrphid Fly Communities in Temperate Fruit Orchard of Kashmir, India. Int. J. Curr. Microbiol. App. Sci., 6(7):2794-2805.

Khan, A.A., Shah, M.A., Majid, S. 2016. Functional response of four syrphid predators associated with green Apple aphid (Hemiptera: Aphididae) in Laboratory. J. Economic Entomol, 109(1): 78-83.

Khan, S.A., Hanif, H. 2016. Diversity and fauna of hoverflies (Syrphidae) in Chakwal, Pakistan. Int. J. Zool. Stud, 1(1):22-23.

Kremen, C., Williams, M.N., Thorp, W.R. 2002. Crop pollination from native bees at risk from agricultural intensification. Proc. Natl. Acad. Sci. U. S. A., 99 (26): 16812-16816.

Leereveld, H., 1982. Anthecological relations between reputedly anemophilous flowers and syrphid flies. III. Worldwide survey of crop and intestine contents of certain anthophilous syrphid flies. Tijdschrift voor Entomologie, 125:25-35.

Margalef, R., 1968. Perspectives in Ecological Theory. University of Chicago Press, Chicago, USA.

Miranda, G.F.G., Young, A.D., Locke, M.M., Marshal,1 S.A., Skevington, J.H., Thompson, F.C. 2013. Key to the genera of Nearctic Syrphidae. Canadian Journal of Arthropod Identification; 23:1-351.

Mitra, B., 2013. Diversity of True flies (Insecta: Diptera) in the estuarine and coastal mangroves of India. (ed: Chapter-4. In Estuaries of India: Biodiversity, Ecology, Conservation and Management, 77-94.

Mitra, B., Bulganin, Mehta, H.S. 2010. A preliminary note on the conservation of Saproxylic flies (Insecta: Diptera) in Himachal Pradesh. Rec Zool Surv India, $110(3): 1-5$.

Mitra, B., Mukherjee, M., Banerjee, D. A. 2008. Check-list of Hover flies (Diptera: Syrphidae) of the Eastern Himalayas. Rec. Zool. Surv. India. Occ. Paper, 284:1-47.

Mitra, B., Parui, P., Banerjee, D., Mukherjeee, M., Bhattacharya, K. A. 2005. Report on flies (Diptera: Insecta) as flower visitors and pollinators of Kolkata and its adjoining areas. Rec. Zool. Surv. India, 105(3-4):1- 20.

Mitra, B., Parui, P., Sharma, R.M. 2004. A preliminary study on the Dipteran flower visitors/pollinators of Himachal Pradesh. Ann. Forestry, 12(1):119-124.

Mitra, B., Roy, S., Imam, I., Ghosh, M. 2015. A review of the hover flies (Syrphidae: Diptera) from India. International Journal of Fauna and Biological Studies. 2(3): 61-73. 
Pape, T., Evenhuis, N.L., Systema Dipterorum, Version [1.5]. 2013. http://www.diptera.org/ [Accessed: 30th January 2015 and 22nd February 2015], 2013.

Potts, S.G., T. Petanidou, S. Roberts, C. O'Toole, A. Hulbert and P. Willemer. 2006. Plantpollinator biodiversity and pollination services in a complex mediterranean landscape. Biol. Conserv., 129: 519-529.

Sajjad, A., Saeed S., Ashfaq, M. 2010. Seasonal Variation in Abundance and Composition of Hoverfly (Diptera: Syrphidae) Communities in Multan, Pakistan. Pakistan J. Zool., vol. 42(2), pp. 105-115,

Shahjahan, M., 1986. Effect of diet on the longevity and fecundity of the adults of the tachinid parasite Trichopoda pennipes pilipes. J. Econ. Ent. 61:11021103.

Shannon, S.E., 1948. A mathematical theory of communication. Bull. Systematic Technology; 27: 379-423.

Shaw, D.C., Taylor, R.J. 1986. Pollination ecology of an alpine fell-field community in the North Cascades. Northwest Science 60:21-31.

Sommaggio, D. 1999. Syrphidae: Can they be used as environmental bioindicators? Agric. Ecosys. Environ, 74:343-356.

Ssymank, A., 2003. Habitatnutzung blütenbesuchender Schwebfliegen (Diptera, Syrphidae) in WaldOffenland-Vegetationsmosaiken.

Berichte der Reinhold-TüxenGesellschaft 15:215-228.

Ssymank, A., Kearns, C.A., Pape, T., Thompson, F.C. 2008 Pollinating flies (Diptera): A major contribution to plant diversity and agricultural production. Biodiversity Issues, 9:86-89.

Talavera, S., Bastida, F., Ortiz P.L., Arista, M. 2001. Pollinator attendance and reproductive success in Cistus libanotis L. (Cistaceae). Int. J. Plant Sci., 162(2): 343-352.

Tooker, J.F., Hauser, H., Hanks, L.M. 2006. Floral host plants of Syrphidae and Tachinidae (Diptera) of Central Illinois. Ann. Entomol. Soc. Am., 99(1):96-112.

\section{How to cite this article:}

Akhtar Ali Khan. 2017. Distribution, Relative Abundance, Species Diversity and Richness of Syrphid Flies in Floricultural Ecosystem of Kashmir. Int.J.Curr.Microbiol.App.Sci. 6(9): 15391552. doi: https://doi.org/10.20546/ijcmas.2017.609.189 